\title{
THE ACTION OF THE OPIUM ALKALOIDS
}

\author{
By F. W. WATKYN-THOMAS, B.A., Trinity College.
}

From the Pharmacological Laboratory, Cambridge

(Received September 9th, 1912)

\section{Previous Work}

Although very complete accounts of the action of morphine have been given by many observers, such as Bernard and Guinard, it is only recently that the action of morphine has been compared with that of the total alkaloids of opium. The action of individual alkaloids, especially narcotine and codeine, has been studied, but the unsuitability of opium for intravenous injection has prevented accurate work on the action of the total alkaloidal content.

In 1909, Sahli ${ }^{1}$ prepared a substance which he called 'Pantopon,' and which contained all the alkaloids of opium in a definite concentration and in soluble form. Since then a great deal of work has been done on the action of this preparation. Loew $y^{2}$ has correlated the action of morphine, codeïne, and the total alkaloids on the respiratory centre. Wertheimer and Raffalovitch ${ }^{9}$ have investigated the action of the total alkaloids on the circulatory system, Rodari, ${ }^{3}$ Bergien, ${ }^{4}$ and Dobeli, ${ }^{5}$ have compared the toxicity of morphine and the total alkaloids. An account of its action is also given by Rodolico, ${ }^{6}$ and Cohnheim has investigated the action on the gut. The analgesic and anaesthetic actions of the total alkaloids have been fully described by $\mathrm{Grey}^{7}$ and Trotain, ${ }^{8}$ among others.

\section{Experimental Methods}

1. Animals. In most cases rabbits were used, as, according to Guinard, in behaviour under morphine they resemble man more closely than do any other animals except the dog. As controls, and in certain experiments for which rabbits were unsuitable, cats were used (Experiments $X, X I I, X I X)$. A few experiments were performed on frogs. (Experiments XVIII, XXIII, XXVII). A certain number of experiments were performed on pithed animals (Experiments II, IV, V, VI, XIX, XXI). 
A.C.E. alone was used in one experiment (XII), A.C.E. and ether in one. In all other experiments the anaesthetic was a 25 per cent. solution of urethane in Ringer, 6 c.c. (1.5 grams) per kilo body weight administered at body temperature. Half the dose was injected under the skin, anaesthesia was induced by ether, and the other half was injected into the peritoneal cavity.

The ether anaesthesia was usually maintained during the preparatory operation, but no other drug was administered until the animal was under the full influence of urethane. It was thus possible to commence all experiments under an approximately standard depth of anaesthesia, as indicated by dosage, blood pressure, size of pupil, and absence of conjunctival reflex.

Tracheotomy was performed, and cannulae tied in the external jugular vein on one side and the common carotid of the other side. Any special operations afterwards performed are described in the account of the experiment. In all experiments injections were given by the cannula in the external jugular; blood pressures were always taken in the common carotid.

Drugs. Tincture of opium is not suitable for experimental work, as the alkaloid content is variable and the contained alcohol is a possible source of error. ${ }^{5}$

Throughout these experiments a 2 per cent. solution of Sahli's 'Omnopon '*-in which all the alkaloids freed from meconic acids, gums and resins are present as hydrochlorides-was used.

The composition of this preparation is as follows:-Morphine 52 per cent., narcotine 20 per cent., codeïne 2 per cent., papaverine 25 per cent., thebaine 1 per cent., narceine $1 \cdot 2$ per cent., other alkaloids 4 per cent., water 8 per cent., hydrochloric acid 9 per cent.

Standard solutions of morphine hydrochloride, narcotine hydrochloride, and codeïne hydrochloride were used. They were prepared on the following basis:-

1 c.c. 2 per cent. omnopon contains 0.0166 gram total alkaloid, 0.0104 gram morphine, 0.0040 gram narcotine, 0.0004 gram codeïne.

1 c.c. standard morphine solution contained 0.0104 gram morphine.

1 c.c. standard narcotine solution contained 0.0040 gram narcotine.

1 c.c. standard codeïne solution contained 0.0004 gram codeïne.

Circulatory effects. The effect of the opium alkaloids on the

* ' Pantopon' is known in this country as 'Omnopon.' I am indebted to Messrs. Hoffman, La Roche, the manufacturers, for a supply of the substance. 
circulation is insignificant. Nevertheless, as there are some pronounced differences between the different bodies, it will be advisable to consider them in detail.

Action on vessels. Perfusion experiments were carried out on the frog, Ringer's solution being injected through the peripheral end of the aorta, and the outflow from the great veins estimated. These experiments showed that morphine in doses of $2-4 \mathrm{mgm}$., thrown suddenly into the circulation-a procedure which gives every advantage to the vessels to show alterations in their calibre-produced practically no effect. If the vessels had been previously acted on by some peripheral vaso constrictor a certain degree of vaso-dilatation was produced. Thus the constriction caused by narcotine could be entirely antagonised by a suitable dose of morphine.

Protocol of typical experiment:Experiment $X X I I$. -Frog B. Average time for outflow of 2 c.c. $=2$ mins. 30 secs.

$4 \mathrm{mg}$. Morphine perfused.

2 c.c. outflow in 2 mins. 25 secs.

2 c.c. outflow in 2 mins. 30 secs.

2 c.c. outflow in 2 mins. 35 secs.

Experiment XXVII.-Frog A. Average time for outflow of 2 c.c. $=59$ secs. $0.4 \mathrm{mg}$. Narcotine perfused.

2 c.c. outflow in 2 mins. 1 sec.

2 c.c. outflow in 4 mins. 2 secs.

$4 \mathrm{mg}$. Morphine perfused.

2 c.c. outflow in 1 min. 12 secs.

2 c.c. outflow in $1 \mathrm{~min} .30$ secs.

2 c.c. outflow in $1 \mathrm{~min}$. 29 secs.

Narcotine in such small doses as $0.4 \mathrm{mgm}$. is shown by this last experiment to produce considerable constriction. This constriction lasts some minutes, and is followed by a very gradual relaxation.

The percentage of morphine in omnopon is, roughly, about the optimum antagonising dose which will annul the constricting action of the contained narcotine. One would anticipate from these experiments that the effect of omnopon on the vessels should not be very pronounced. This was found to be the case : omnopon producing little effect in either direction.

Protocol of typical experiment:-

Experiment XXII.-Frog A. Average time for outflow of 4 c.c. $=1 \mathrm{~min} .5$ secs.

$7 \mathrm{mg}$. Omnopon perfused.

4 c.c. outflow in 1 min. 9 secs.

4 c.c. outflow in 1 min. 6 secs.

4 c.c. outflow in 1 min. 5 secs. 
Action on the heart. None of these alkaloids produce any decided action on the heart in the intact animal in dosage which may be regarded as therapeutically possible. In the isolated mammalian heart, where concentrated solutions can be suddenly thrown into the coronary circulation, it is possible to make comparative experiments on their toxicity. By this means it was shown that $2 \mathrm{mgm}$. morphine produced little or no effect, whilst $5 \mathrm{mgm}$. caused decided slowing and diminished systole lasting for many minutes, in spite of the fact that the morphine passed straight through in less than one minute and was then replaced by normal Ringer's fluid.

Narcotine in doses up to $1 \mathrm{mgm}$. left the heart entirely uninfluenced.

Omnopon in doses up to $2 \mathrm{mgm}$. causes some increase in the systole, but beyond this dose the systole weakens, and if a dose of $4 \mathrm{mgm}$. is injected into the coronary circulation weakening of systole and slowing of rate are always pronounced, while the beat may be inhibited for thirty to sixty seconds.

Measurements of the output of the mammalian heart in the intact and anaesthetised animal show that none of these alkaloids, under such conditions, exert any appreciable action on the heart, even in poisonous doses.

It is true that a dose, sufficient to cause a fall of pressure by vasodilatation, causes an increased output from the heart, but this is not the direct effect of the alkaloids but is associated with the vaso-motor fall, and may be produced equally well by suitable doses of some of the nitrites.

Protocol of experiment:-

Experiment $X$.-Cat 2 kilos. Ether and urethane. Tracheotomy. Cannulae in external jugular and common carotid. Artificial respiration, thorax opened and heart in cardiometer.

$\begin{array}{lllllll}1 \text { c.c. Morphine } & \ldots & \ldots & \ldots & \ldots & \ldots & \text { Slight fall of blood pressure. Some variation }\end{array}$ in beat of heart.

1 c.c. Omnopon ... $\quad \ldots \quad \ldots \quad \ldots \quad \ldots$ Fall of pressure. No effect on heart.

3 c.c. Narcotine ... $\quad \ldots \quad \ldots \quad \ldots \quad \ldots \quad$ Fall and rise to previous pressure. No effect on heart.

$\begin{array}{lllllll}3 \text { c.c. Narcotine } & \ldots & \ldots & \ldots & \ldots & \ldots & \text { Fall of pressure. No effect on heart. }\end{array}$

$\begin{array}{llllllll}3 \text { c.c. Narcotine ... } & \ldots & \ldots & \ldots & \ldots & \text { Fall of pressure. Weakening of heart. }\end{array}$

4 c.c. $0.25 \%$ Nicotine $\ldots \quad \ldots \quad \ldots \quad \ldots \quad$ No rise of pressure. Heart growing rapidly weaker.

Death.

In none of my experiments has the slightest evidence been afforded that any of these opium alkaloids, or all of them administered together as omnopon, exert any direct action on the heart. 
Blood pressure. The action of morphine on the blood pressure is insignificant. Many writers claim that it induces a rise of pressure, and it is true that small doses, injected directly into the circulation, may cause an increase in pressure of a few millimetres of mercury. But I have never obtained the smallest rise by subcutaneous injection. Larger doses, such as $5 \mathrm{mgm}$. per kilo body weight, in rabbits undoubtedly produce a small degree of vaso-dilatation and some corresponding fall in blood pressure, but it is only by the administration of enormous doses, $10 \mathrm{mgm}$. per kilo, that a fall of pressure of any moment can be produced. It is hardly necessary to point out that in all experiments in which such bodies as these are injected efficient respiration is an essential for accurate estimation of the effect produced.

Codeïne has more action on blood pressure than morphine, but its effect is still insignificant. It raises the height of the blood pressure curve a shade more than morphine, and it is much easier with this alkaloid to produce a marked fall in pressure associated with vaso-dilation. Codeïne stands midway in its action between morphine and narcotine.

Narcotine, in small doses, causes a distinct rise in arterial blood pressure, but when the dosage is increased fall of pressure rapidly ensues. The rise in pressure is associated with vaso-constriction, and it is easy to show that the vessels of the gut constrict more or less in proportion to the rise of pressure. In the same way the subsequent fall in pressure is associated with splanchnic vaso-dilation. Now, as narcotine is representative of a number of other alkaloids present in opium which in large doses produce fall of pressure and in small doses initial constriction, it will be well to examine the mechanism by which this type of effect is produced.

A large dose of narcotine injected into the circulation immediately produces a maximal fall in blood pressure. Further injections produce no further fall, although it may readily be shown that the blood pressure is capable of a further fall by the administration of a little nitrite. If, after the pressure has undergone this maximal fall, which may occur in a rabbit after the injection of $15 \mathrm{mgm}$. per kilo body weight, the splanchnic nerve be excited by a faradic current, no response-vasoconstriction and rise of blood pressure-occurs. Nevertheless, such response may be obtained by applying the electrodes to the postganglionic fibres. The splanchnic nerve is paralysed and the block occurs in the ganglion cells. This, then, is the explanation of the fall of blood pressure caused by these alkaloids. The sympathetic ganglion cells in the 
neck may be paralysed in a similar manner. Using dilatation of the pupil as a test of sympathetic activity, it was found that narcotine paralysed this nerve and that the block occurred in the ganglion cells, the pupil reacting normally to stimulation of the post-ganglionic fibres.

Protocol of experiment:-

Experiment XIII.-Rabbit 1.7 kilos. Ether and urethane. Tracheotomy. Cervical sympathetic exposed. Electrodes between mid- and superior ganglia.

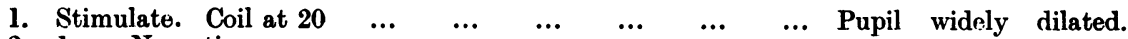

2. 1 c.c. Narcotine.

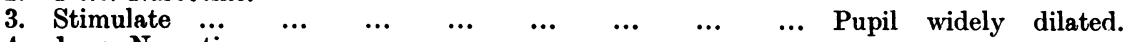

4. 1 c.c. Narcotine.

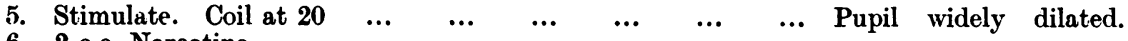

6. 2 c.c. Narcotine.

7. Stimulate. Coil at $20 \quad \ldots \quad \ldots \quad \ldots \quad \ldots \quad \ldots \quad \ldots \quad \ldots$ Pupil dilated, but less.

8. 3 c.c. Narcotine.

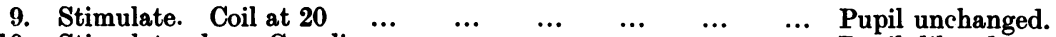

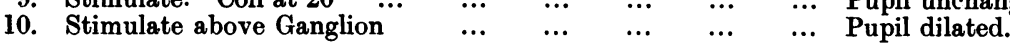

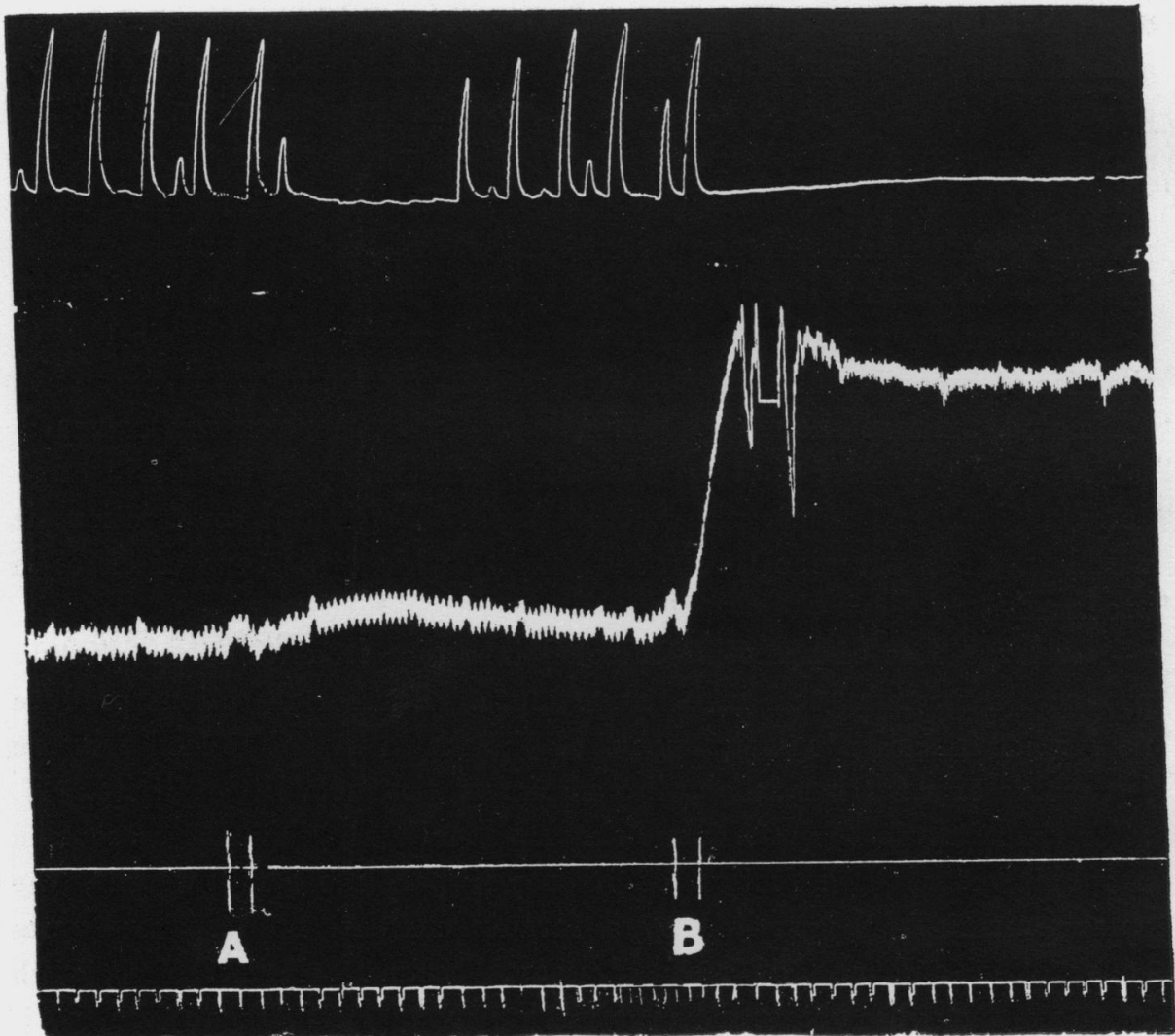

Fig. I.-Cat, urethane. Intestinal movements recorded by balloon. Blood pressure below. At $A-1$ c.c. $0.5 \%$ Nicotine injected.

At B-2 c.c. $0.1 \%$ Adrenalin. 
In other words, narcotine and its allies should produce their rise, and more pronounced fall in blood pressure, in the same manner as nicotine. The first action of nicotine is to stimulate very powerfully the ganglion cells of the sympathetic system, and, if these are paralysed by narcotine, nicotine should produce no action, which was found to be the case; whilst adrenalin, which is known to act on the end organ, produces its action almost uninfluenced. Two or three points in this type of paralysis are worthy of note. The first is that the paralysis may occur even whilst the medulla is still active, as is shown by natural respiration. This is a remarkable fact, and, so far as I am aware, every other paralysis of this nature-such as that caused by nicotine or by apocodeine-is preceded by complete medullary paralysis.

Protocol of experiment :-

Experiment XI-Rabbit 1.9 kilos. Ether and Urethane. Tracheotomy. Cannulae in Ext. Jugular and Common Carotid.

1. 2 c.c. Narcotine.

2. 2 c.c. Narcotine.

3. 1 c.c. Narcotine.

4. 1 c.c. Narcotine.

5. 2 c.c. Adrenalin $\ldots$ Marked effect.

6. 2 c.c. Nicotine _.. No effect. Feeble respiratory movements continuing.

Secondly, the nerve cells on the course of the splanchnic fibres supplying the vessels of the gut are paralysed before those conveying inhibitory fibres to intestine. Thus an injection of nicotine may fail to cause any rise in blood pressure, and yet inhibit peristalsis (vide protocol, Expt. XII).

Omnopon certainly raises blood pressure more than either morphine, codeine, or narcotine, in corresponding doses. In the rabbit, doses of over $15 \mathrm{mgm}$. per kilo body weight cause a marked fall of pressure with all the characteristics of that described for narcotine, and in sufficient doses will, like narcotine, entirely antagonise the pressor action of nicotine. This is in contradiction to the results of Bergien who claims that blood pressure is not materially affected by large doses of omnopon.

The principal fact learnt from these experiments is that the opium alkaloids exert an initial slight stimulant action on the sympathetic cells, this being followed by depression and paralysis. This is very marked in the case of narcotine, much less for codeïne, and least of all for morphine.

Comparison of Respiratory Effects. A requirement in medicine is a drug which, while diminishing cerebral reflexes like morphine, will do 
so without marked depression of respiration. This is especially desirable in the treatment of useless cough. Morphine, unfortunately, profoundly depresses the respiratory centre, no doubt by preventing afferent impulses reaching the medulla.

Codeïne, heroine, dionine, and other morphine derivatives have been employed as substitutes, and it is generally admitted that they are less depressant to respiration than morphine, while it is stated that they are efficient in relieving cough.

Loewy $^{2}$, Wertheimer and Raffalovich ${ }^{9}$ and Rodari ${ }^{3}$ claim that the total alkaloids have considerably less effect on the respiratory centre than has morphine.

Loewy has shown that the reaction of the respiratory centre to carbon dioxide is less affected by the total alkaloids than by morphine.

All these observers used 'omnopon.'

In the ensuing experiments the effect on the respiratory reflexes was tested by stimulation of the cut central end of the great sciatic, or of the external popliteal, with induction shocks and a tetanising current. The respiratory movements were recorded by a lever attached to the severed ensiform cartilage.

Protocols of typical Experiments :-

Experiment XVII.-Rabbit, 1.30 kilos. Ether and urethane. Tracheotomy. Cannulae in left external jugular. Right common carotid. 'Diaphragm slip' method of recording great sciatic on electrodes.

i. Stimulate great sciatic. Coil at $20 \quad \ldots \quad$ (a) Great rise of blood pressure. TraubeHering curves.

(b) Respiration greatly increased in force and frequency.

ii. 0.1 c.c. Omnopon $\quad \ldots \quad \ldots \quad \ldots \quad($ a $)$ Slight rise of blood pressure.

(b) Respiration deepened, but not appreciably slowed.

iii. 0.2 c.c. Omnopon $\quad \ldots \quad \ldots \quad \ldots \quad(a)$ Further rise of blood pressure

(b) Respiration slightly slower.

iv. Stimulate great sciatic. Coil at 20 ... Respiratory reflex rery feeble.

v. Stimulate great sciatic. Coil at 15 ... Vomiting movements. Traube-Hering waves. Rise of blood pressure.

vi. 1 c.c. Morphine $\quad \ldots \quad \ldots \quad \ldots \quad \ldots$ Respiration slowed and abolished. Drum stopped for artificial respiration.

vii. Stimulate great sciatic. Coil at 15

Vomiting movements followed by increased frequency.

viii. $\mathbf{0 . 3}$ c.c. Narcotine

... Fall in blood pressure. Return to Normal. Respiration more rapid.

ix. Stimulate great sciatic. Coil at 15 ... Response good.

x. $\mathbf{0} \cdot \mathbf{6}$ c.c. Narcotine $\quad \ldots \quad \ldots \quad \ldots \quad$ Fall of blood pressure. Arrest of respiration. Death. 
Experiment XVIII-Rabbit, 1.5 kilos. Anaesthetics and operative procedure, as No. XVII.

i. Stimulate great sciatic. Coil at 35 ... Rise of blood pressure. Respiration deeper and more rapid.

ii. 0.2 c.c. Omnopon $\quad \ldots \quad \ldots \quad \ldots \quad$ Rise of blood pressure. Respiration unaffected at first, and then more shallow.

iii. Stimulate great sciatic. Coil at $35 \quad \ldots \quad$ No effect on blood pressure or on circulation.

iv. 0.2 c.c. Omnopon $\quad \ldots \quad \ldots \quad \ldots$ Rise of blood pressure. Respiration unaffected.

v. Stimulate great sciatic. Coil at $25 \quad \ldots$ Slight rise of blood pressure. No respiratory response.

v. Wash out-wait three minutes.

vii. $0 \cdot 2$ c.c. Morphine $\quad \ldots \quad \ldots \quad \ldots$ Very slight rise in blood pressure. Respiration unaffected.

viii. Stimulate great sciatic. Coil at 35 ... Absolutely no response in blood pressure or circulation.

ix. Stimulate great sciatic. Coil at 25 ... Absolutely no response in blood pressure or circulation. $\begin{array}{lllll}\text { x. } 0.2 \text { c.c. Narcotine } \quad \ldots & \ldots & \ldots & \text { Fall of blood pressure. Rate of respiration } \\ \text { increased. }\end{array}$

xi. Stimulate great sciatic. Coil at 25 ... Rise of blood pressure. Respiration unaffected.

It was found that morphine always slows respiration and depresses the reflexes. Narcotine at first renders respiration deeper and slower, and if the drug is pushed the movements become more rapid and more shallow. But the most remarkable effect of narcotine on respiration is its depression of the respiratory reflexes.

It becomes necessary to explain the paradox as to how respiration, which is largely a reflex phenomenon, can be stimulated by narcotine whilst the sensory reflexes are depressed. Taken in conjunction with the work of Loewy this suggests that in the respiratory centre there is a different receptive mechanism inducing respiratory activity for chemical afferent nervous stimuli. One explanation which might be offered to account for the difference in the action of morphine and narcotine would be that, while morphine depresses both mechanisms, narcotine only depresses the one; or it may be that the medulla is rendered hypersensitive to carbon dioxide and other chemical stimuli acting directly on its motor mechanism, whilst afferent stimuli may still be blocked on the sensory side.

Omnopon invariably increased the depth of respiration without materially altering its rate. This effect is not very marked, although quite distinct. Large doses, $15 \mathrm{mg}$. per kilo body weight in a rabbit, 
cause a temporary paralysis of respiration. As compared with morphine, then, omnopon must be regarded as less toxic, and much less depressant to respiration.

Nevertheless, it decidedly depresses the reflex response to nervous stimulation, and, therefore, should be greatly superior to morphine in the treatment of cough.

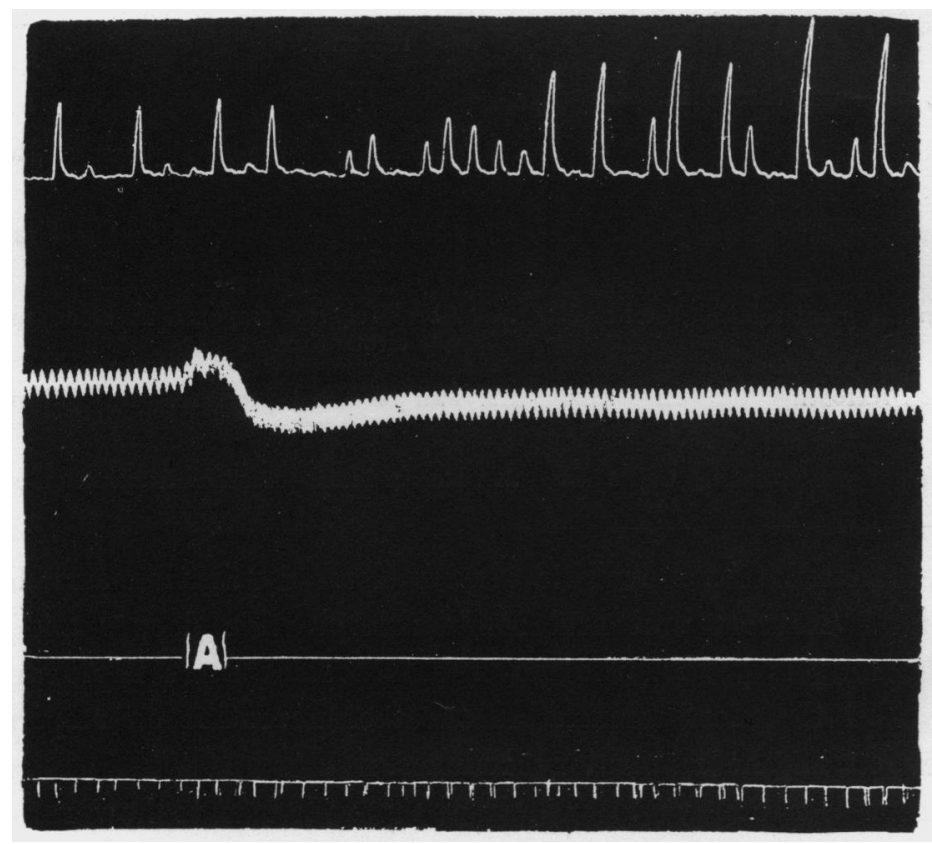

Fra. II.-Cat. Urethane. Intestinal movements and blood pressure. Showing effect of a late injection of 2 c.c. Omnopon at A.

Intestinal Movements. It is well known that morphine, probably during its excretion, influences Auerbach's plexus in such a way as to diminish peristaltic movement. If, however, a large dose of morphine is suddenly thrown into the circulation the first effect is invariably augmented peristalsis, which in the case of man may lead to diarrhoea and vomiting. Narcotine shows this effect in a very much more pronounced way than morphine. This drug exerts no stimulant action on any form of plain muscle or motor nerve ending, but it has a markedly paralysing action on sympathetic ganglia; hence, it is not unreasonable to suppose that this action is due to depression of the ganglia on the course of the inhibitory nerves to the intestine. 
Omnopon, like narcotine, at first increases peristalsis. For the time being, the narcotine overshadows the morphine action. This effect is, of course, followed by diminished peristalsis. But, whether this explanation be correct or not, the important fact remains that omnopon causes at every stage of its action much less effect in diminishing peristalsis than morphine.

Protocol of typical experiment:-

Experiment XII.-Cat, 2.5 kilos. A. C. E. Tracheotomy. Cannulae in left common carotid and right external jugular. Abdomen opened. Incision made in a loop of small intestine, and balloon inserted.

(a) 2 c.c. 'Omnopon' ... $\quad \ldots \quad \ldots \quad \ldots$ Great fall in blood pressure. Slight increase in peristalsis.

(b) 3 c.c. 'Omnopon' ... $\quad \ldots \quad \ldots \quad \ldots$ Very little effect on gut movements at first, Artificial respiration.

Wash out.

Wait for respiration to become natural.

(c) 2 c.c. ' Omnopon' ... $\quad \ldots \quad$.. $\quad \ldots$ No effect on blood pressure. Gut movements further increased. Respiration natural.

(d) 6 c.c. $0.5 \%$ Narcotine $\quad \ldots \quad \ldots \quad \ldots$ Great increase in gut movements. Temporary Artificial respiration. cessation of respiration.

Respiration natural.

(e) 1 c.c. $0.5 \%$ Nicotine .. $\quad . \quad \ldots \quad$ No effect on blood pressure. Temporary

(f) 2 c.c. $0 \cdot 1 \%$ Adrenaline

Great rise in blood pressure. Cessation of peristalsis. 


\section{REFERENCES}

I. Sahli, Therapeut. Monatshe|te, IX, p. 1, 1909 ; Munchener Med. Wochen., LVII, p. 1326, 1910.

2. Loewy, Munchener Med. Wochen., LVIII, p. 2408, 1910.

3. Rodari, Therapeut. Monatshefte, X, p. 540, 1910.

4. Bergien, Munchener Med. Wochen., LVIII, p. 2409, 1910.

5. Dobeli, Monats. f. Kinderheilkunde, IX, p. 34, 1910.

6. Rodolico, Giornale internaz. d. Scienze Mediche., Ann. XXXII, 1910.

7. Grey, Lancet, p. 673, 1911.

8. Trotain, L'Etude Therapeutique de lopium, Paris, 1911.

9. Wertheimer and Raffalovitch, Deut. Med. Wochen., XXXVII, p. 1710, 1910. 